\title{
1 How to build a mycelium: tradeoffs in fungal architectural traits
}

2

3

4 Anika Lehmann 1,2, ${ }^{*}$, Weishuang Zheng 3 , Katharina Soutschek 1, Matthias C.

5 Rillig 1,2

6

71 Freie Universität Berlin, Institut für Biologie, Plant Ecology, Altensteinstr. 6, D-14195

8 Berlin, Germany;

92 Berlin-Brandenburg Institute of Advanced Biodiversity Research (BBIB), D-14195 Berlin,

10 Germany;

113 State key Laboratory of Microbial Technology, Shandong University, Qingdao 266237,

12 China

13

14 * Corresponding author, Freie Universität Berlin, Institut für Biologie, Plant Ecology,

15 Altensteinstr. 6, D-14195 Berlin, Germany. Tel.: +49 30 83853145. Fax: 493083853886.

16 E-mail address: lehmann.anika@googlemail.com

17

18 Keywords: saprobic fungi, traits, tradeoff, mycelium, architecture 


\section{Abstract}

21

22 The fungal mycelium represents the essence of the fungal lifestyle, and understanding how a

23 mycelium is constructed is of fundamental importance in fungal biology and ecology.

24 Previous studies have examined initial developmental patterns or focused on a few strains,

25 often mutants of model species, and frequently grown under non-harmonized growth

26 conditions; these factors currently collectively hamper systematic insights into rules of

27 mycelium architecture. To address this, we here use a broader suite of fungi (31 species

28 including members of the Ascomycota, Basidiomycota and Mucoromycotina), all isolated

29 from the same soil, and test for ten architectural traits under standardized laboratory

30 conditions.

31 We find great variability in traits among the saprobic fungal species, and detect several clear

32 tradeoffs in mycelial architecture, for example between internodal length and hyphal

33 diameter. Within the constraints so identified, we document otherwise great versatility in

34 mycelium architecture in this set of fungi, and there was no evidence of trait 'syndromes' as

35 might be expected.

36 Our results point to an important dimension of fungal properties with likely consequences for

37 coexistence within local communities, as well as for functional complementarity (e.g.

38 decomposition, soil aggregation). 


\section{Introduction}

42

The mycelium comprises the entirety of the hyphae of a fungus, representing its nutrientcapture and interaction interface, and the infrastructure for transport within the fungal individual. This structure is designed for a dynamic exploratory lifestyle with its ability to reconfigure, fragment and fuse, and represents the very essence of the fungal lifestyle ${ }^{1}$. Understanding how a mycelium is built is therefore of fundamental importance for gaining insight into fungal biology and ecology.

The initial development of the mycelium starting from germinating spores has been extensively studied (e.g. ${ }^{2,3}$ ), revealing some general hyphal growth patterns: emerging from a spore, a hypha extends at an exponential rate followed by a constant linear phase until the formation of a new branch is initiated; each new branch itself follows this exponential-linear phase pattern. Additionally, hyphae show negative autotropism and radial orientation away from the colony center ${ }^{3}$, eventually giving rise to the characteristic circular (in 2D) or spherical (in 3D) shape of 'colonies' or fungal individuals.

Mycologists have also examined the kinetics and branching behavior of fungi with a focus on a limited suite of traits and with a typical focus on mutants of the same species or a few species, mainly derived from fungal culture collections (e.g. $\left.{ }^{4-6}\right)$. These investigations revealed that fungal mycelia undergo changes in growth behavior due to differentiation. Studies on Neurospora crassa revealed that after approximately $22 \mathrm{~h}$ branching angles decrease while hyphal extension rate and diameters increase. Ultimately, the mycelium establishes a hierarchy in which hyphae of higher branching order have decreased hyphal growth rate and diameter in relation to the parental hyphae from which they emerged. As a consequence, the space-filling capacity of the mycelium increases ${ }^{7}$, leading to a maximum surface area while investing in a minimum of hyphal length ${ }^{8}$.

Apart from such studies of development, there is no systematic comparison of a range of architectural features, measured under the same, standardized laboratory conditions, on a larger set of fungi from a common ecological context. This is why we currently only have limited knowledge about tradeoffs governing mycelium architecture that could give insight on architectural 'rules'. So far, insights into such tradeoffs come from studying single species or mutants, and this frequently has not resulted in a consensus. For example, the relationship between hyphal diameter and growth rate can be positive (e.g. Bortrytis) or neutral (e.g., Mucor strains) ${ }^{9}$. The same discrepancy holds true for the relationship between hyphal branching frequency (number of hyphal tips) and hyphal growth rate, which was examined in 
multiple studies focusing on Neurospora strains and mutants: some studies found a positive relationship ${ }^{10}$ and others did not ${ }^{11}$.

Recently, advantages of pursuing a trait-based approach in fungal ecology have been introduced $^{12,13}$. One clear benefit of such an approach is to move beyond idiosyncratic comparisons of a few isolates to making comparisons using larger sets of fungal isolates, offering opportunities for more general inferences about mycelium architectural rules. Here, we report on studies designed to collect mycelium architecture traits for a set of 31 saprobic fungal strains, containing members of the phyla Ascomycota, Basidiomycota and Mucoromycotina. Our goal was to uncover general 'rules' of mycelium construction by identifying tradeoffs among mycelium growth characteristics within this set of fungi, all isolated from the same soil.

\section{Materials and Methods}

Fungal strains. Fungal strains were originally cultured from Mallnow Lebus, a dry grassland in a nature conservation reserve (Brandenburg, Germany, 52²7.778' N, 14²9.349' E). A set of 31 fungal strains were isolated from soil samples as described in Andrade-Linares et al. ${ }^{14}$. Briefly, soils were diluted or washed to minimize spore abundance and increase the isolation of fungi derived from hyphae attached to soil particles ${ }^{15,16}$. For isolation a variety of media and antibiotics were used to target Ascomycota, Basidiomycota and Mucoromycotina while suppressing bacterial growth. Isolates were incubated at $22^{\circ} \mathrm{C}$ and were cultured on PDA. The fungal set comprised members of the Ascomycota (twenty strains), Basidiomycota (four strains) and Mucoromycotina (seven strains) (Fig.1a, Table S1).

Architecture traits. We conducted two separate studies to collect architectural traits for the 31 fungal strains. All studies were performed in vitro with PDA as growth substrate. In the first study, we focused on measuring hyphal branching angle (BA), internodal length (IL) and diameter (D). For this, the fungal strains were grown on single concavity slides carrying 150 $\mu \mathrm{l}$ of PDA. We chose to reduce the concentration of PDA to $10 \%$ to obtain nutrient reduced growth medium for reduced mycelial density. This was necessary to be able to identify single hyphae in very densely growing fungi. To guarantee solidification of the medium, we added agar (Panreac AppliChem) to reach $15 \mathrm{~g} \mathrm{~L}^{-1}$ concentration. The growth medium was flattened by placing a cover slip on the liquid medium drop until it solidified. A pre-sterilized poppy seed carrying the target fungal strain was positioned in the center of the concavity. The slide was placed in a $9 \mathrm{~cm}$ Petri dish filled with a $5 \mathrm{~mm}$ layer of water agar to maintain high air humidity. Plates were sealed and stored at room temperature $\left(22^{\circ} \mathrm{C}\right)$ in the dark until the 
111 fungal colony covered half of the concavity area. For each fungal strain five slides were

112 prepared and placed in separate Petri dishes. For the measurements, slides were examined

113 under the microscope (Leica DM2500, bright field, 200x). Per slide, we randomly chose five

114 hyphae as subsamples; for each of these hyphae we measured at the colony edge the last

115 developed branching angle, the internodal lengths between this last and second-to-last

116 branch and the hyphal diameters within this youngest internodal segment. For analyses, we

117 used the image processing software Image $J^{17}$. For each experimental unit, we calculated a

118 mean value and a coefficient of variation (CV) from the subsample data. These represented

119 two aspects of a trait: the average value and its variability. The trait data used in statistical

120 analyses were the average of mean values and CVs of five replicates (i.e. $n=31$ ).

In the second study, we investigated the complexity and the heterogeneity of fungal mycelia by applying fractal analysis - a technique used to assess self-similarity and space-filling capacity of fungal hyphae ${ }^{18}$. For this, we applied the same approach as in the first experiment but with eight replicates per fungal strain. At harvest, the slides were examined under the microscope (Leica DM2500, bright field, 200x) focusing on the outer $200 \mu \mathrm{m}$ of the growing zone to investigate the "surface fractals" chosen to generate grayscale photos with high contrast (background: white, hyphae: black; Fig.1b). For each slide, we photographed three fields of view at the colony edge. These settings and further image processing in Image $J^{17}$ and Adobe Illustrator (CS6, v.16.0.0) were necessary to guarantee comparable and unbiased photos that can be processed by image analysis software. First, photos were converted to 8-bit binary images in ImageJ and subsequently hyphae were skeletonized. For this, a thinning algorithm repeatedly reduced pixels from the edge of the target object until a one-pixel wide shape was reached ${ }^{20}$. In Illustrator, the skeletonized hyphae were reconnected and image artifacts excluded, if necessary. Line thickness was adjusted to mean diameter trait values derived from experiment one. The final processed images were loaded into the ImageJ plug-in "FracLac" to measure fractal dimensions. We chose box counting dimensions (Db) as a measure of structural complexity (i.e. the degree of detail or amount of parts a pattern consists of), and lacunarity $(\mathrm{L})$ as a representative of structural heterogeneity (i.e. the gappiness or "rotational and translational invariance" in a pattern $\left.{ }^{21}\right)$. We used default settings but allowed for rotational orientations in analyses. Finally, subsample data were used to calculate CVs for box counting dimension and lacunarity. The subsample data were then merged to one mean and CV trait value per replicate. Additionally, we verified if implementing diameter data altered fractal dimension data by correlating skeletonized and adjusted diameter data for both box counting dimension and lacunarity (Fig. S1). 
148 Statistics. We analyzed the relationships between the ten architectural trait variables

149 derived from 31 saprobic fungal strains represented by both mean value and coefficients of

150 variation $(\mathrm{CV})(\mathrm{n}=31)$. First, to evaluate fungal distribution in ten-dimensional trait space, we

151 ran a principal component analysis using the function $\operatorname{prcomp}()$ in the package "stats" with z-

152 transformed data. Significance of PC axes was determined via the function testdim( ()$^{22}$ in the

153 package "ade4"23-25. Only the first axis was significant, hence we included PC axis 1 and 2 in

154 the visualizations without losing information from the excluded axes. Next, we conducted

155 kernel density estimation to assess species occurrence probability following the procedure

156 presented by Diaz et al. ${ }^{26}$. Briefly, we used kde() function of the "ks" package ${ }^{27}$ with

157 unconstrained bandwidth selectors by implementing the function $\mathrm{Hpi}($ ) on our first two PC

158 axes. Using the function contourLevels() we estimated contour probabilities for 0.5 and 0.95

159 quantiles.

160 Second, to test for phylogenetic signal in our trait variables we used Moran's I statistic, a

161 measure for phylogenetic autocorrelation, as implemented in the package "phylosignal". We

162 accounted for phylogenetic relatedness among species (indicated by detected phylogenetic

163 signals) by calculating phylogenetically independent contrast of our trait variables with the

164 packages "picante"28 and "ape" ${ }^{29}$ using the functions pic() and match.phylo.data(). We

165 evaluated if the assumptions of the Brownian motion model were satisfied by our data ${ }^{30}$. For

166 that, we investigated the standardization of the contrasts via diagnostic regression tests to

167 evaluate the relationship between absolute standardized contrasts and (i) the square root of

168 their standard deviation ${ }^{31}$ and (ii) the node height (i.e. node age ${ }^{32,33}$ ). Identified influential

169 nodes were excluded, following the threshold of absolute studentized residuals greater than

$170 \quad 3^{31,34}$. To satisfy the Brownian motion model assumption, we used log transformed trait

171 values und excluded two outlier nodes (node 49 and $61^{35}$ ).

172 Third, multiple pairwise correlations using Pearson's rho were conducted and plotted with the

173 function corrplot() in the eponymous package ${ }^{36}$. Analyses were done for original (non-

174 transformed, $n=31$ ) and phylogenetically corrected data (log-transformed, $n=28$ ).

175 Fourth, we ran linear regressions and further investigated the relationships by quantile

176 regression with the package "quantreg" (https://github.com/cran/quantreg). Under most

177 ecological conditions, linear regressions tend to over- or underestimate relationships due to a

178 focus on the mean of the response distribution. Especially in wedge-shaped data

179 distributions, indicating unmeasured limiting factors, quantile regressions are more

180 informative since they test the relationship between response and predictor variable at their

181 maxima $^{37,38}$. Both regression analyses were run on z-transformed data and model residuals

182 were tested for homogeneity and normal distribution. Additionally, we ran multiple pairwise

183 regressions for both original and phylogenetically corrected data to provide graphical 
184 information on data distributions of all trait combinations (see Fig. S2 and S3). These were

185 generated by the function ggpairs() of the package GGally ${ }^{39}$.

186 All analyses were conducted in R (v. 3.4.140) and plots were created with the graphics

187 package ggplot ${ }^{41}$ and its extension GGally.

189 Results and discussion

190 Trait expression. Overall, we found high variability among strains for all traits (Fig.1). The

191 application of fractal dimensions on mycelium structure revealed that trait mean values of

192 box counting dimensions $(\mathrm{Db})$ ranged between 1.2 and 1.6, where a value of 1 represents a

193 single unbranched hypha, and a value of 2 a complex space-filling mycelium. The most

194 complex mycelium was found in the Mucoromycotina, while Basidiomycota had the most

195 simply structured mycelia. For lacunarity (L), we found in our study that trait values ranged

196 between 0.4 (Basidiomycota) and 0.7 (Ascomycota). With increasing trait value, the

197 heterogeneity and hence gappiness of the mycelium increased. The investigation of hyphal

198 features revealed that the branching angle (BA) varied substantially across fungal strains

199 from 26 to $86^{\circ}$ with Mucoromycotina having large angles and Basidiomycota rather small

200 angles. For hyphal diameter (D) trait values ranged from 2.7 to $6.5 \mu \mathrm{m}$ across the 31 strains

201 where both extremes could be found in the Mucoromycotina. The length of the hyphal

202 internodes (IL) showed considerable differences: Within Basidiomycota internodal lengths of

$203453 \mu \mathrm{m}$ could be reached while in Mucoromycotina the lowest value of $40 \mu \mathrm{m}$ was measured.

204 Our values are within the range of previously reported architectural features of selected,

205 individual saprobic filamentous fungi (e.g. $\left.{ }^{19,42-46}\right)$.

206 After establishing the trait database, we investigated the trait space generated by the

207 collected fungal architectural features. To do this, we applied principal component analyses.

208

209 PCA. For our 31 fungal strains, the sole significant first principal components accounted for

$21034 \%$ of the variability in the ten architecture traits (Fig. 2a). In this ten-dimensional trait

211 space, the set of our 31 fungal strains occupied the whole PC plane with a clear separation

212 of the three phyla across the plane. Considering the sole significant PC axis 1, Ascomycota

213 assembled in the center flanked by Mucoromycotina on the left, driven by large branching

214 angles and high mycelial complexity, and Basidiomycota on the right portion, primarily

215 characterized by long internodes and wide hyphal diameters (Fig. S4). Across species, some

216 clear correlations among traits became visible; hence, we further investigated the type and

217 intensity of potential architectural tradeoffs for our fungal set. 
219 Phylogenetic signal. For this, we first tested all ten traits for a phylogenetic signal to

220 evaluate if the phylogenetic relatedness among fungal strains can influence any trait

221 relationships we want to investigate. Applying Moran's I statistics (Table S2), we found

222 phylogenetic signals in $\mathrm{Db}(\mathrm{I}=0.16, \mathrm{p}=0.02)$ and $\mathrm{L}_{\mathrm{cv}}(\mathrm{I}=0.13, \mathrm{p}=0.02)$. Hence, we needed to

223 account for phylogenetic relations for these two traits among our 31 fungal strains by

224 applying phylogenetically independent contrast in the following analyses.

225

226 Tradeoffs. We found 14 trait pairs with significant correlations of which ten passed

227 phylogenetic correction (Fig. 2b, Fig.S2 and S3). The strongest correlations were detected

228 between mycelium complexity and its coefficient of variation ( $\mathrm{Db}-\mathrm{Db}_{\mathrm{cv}}$ in Fig.2c), mycelium

229 heterogeneity, as measured by lacunarity, (Db - L in Fig.2d) and hyphal diameter (Db - D in

230 Fig.2e), as well as between branching angle and its coefficient of variation (BA - BA $A_{C V}$ in

231 Fig.2f). For internodal length, we detected relationships with mycelium complexity (IL - Db in

232 Fig.2g), variability in mycelium complexity (IL - Db $b_{\mathrm{cv}}$ in Fig.2g) and hyphal diameter (IL - D in

233 Fig. 2i). Another strong correlation was found between the coefficients of variation of

234 mycelium complexity and branching angle ( $\mathrm{Db}_{\mathrm{cv}}-\mathrm{BA}_{\mathrm{cv}}$ in Fig.2j). In addition, weak

235 correlations were found for $\mathrm{D}$ and $\mathrm{Db}_{\mathrm{cv}}, \mathrm{D}$ and $\mathrm{L}_{\mathrm{cv}}$, as well as between IL and $\mathrm{L}_{\mathrm{cv}}$, IL and $B A$

236 (Fig.S2 and S3). From these correlations we can deduce multiple rules for mycelium

237 architecture.

238 For structural complexity (as represented by box counting dimensions) and branching angle

239 we detected a negative relationship between their mean values and $\mathrm{CVs}\left(\mathrm{Db}-\mathrm{Db}_{\mathrm{CV}}\right.$ in Fig.

$2402 \mathrm{C}$ and $\mathrm{BA}-\mathrm{BA}_{\mathrm{cv}}$ in Fig. 2f). Thus strains exhibiting a high trait value for $\mathrm{BA}$ or $\mathrm{Db}$ are

241 restricted to this high value, while strains with low values in these traits are capable of further

242 adjusting these features.

243 Within strains, variability in mycelial complexity itself is determined by increasing internodal

244 length (IL $-\mathrm{Db}_{\mathrm{CV}}$ in Fig. $2 \mathrm{~h}$ ) and higher flexibility in branching angle measures $\left(\mathrm{Db}_{\mathrm{CV}}-\mathrm{BA}_{\mathrm{CV}}\right.$ in

245 Fig. 2j). Thus, the degree of mycelial complexity can be modulated via branching patterns

246 (e.g. distance between branches).

247 Considering the complexity - the space-filling capacity - of a mycelium, we found that more

248 complex mycelia are more heterogeneously structured (Db - L in Fig.2d). Mycelia with high

249 space-filling capacity tend to be rather heterogeneous in their structure, i.e. their mycelium is

250 not uniformly complex but rather exhibits complex zones replaced by more simple mycelium

251 structures towards the growing edge. At the colony edge, hyphae are confronted with new

252 resources and environmental conditions for which a maximum of flexibility is likely

253 advantageous. Furthermore, complex mycelia have smaller hyphal diameters (Db - D in Fig.

$2542 \mathrm{2e}$ ) and shorter internodal length (IL - Db in Fig.2g). A mycelium with long internodes is

255 characterized by less branching and hence less space-filling. However, to be capable of 
256 growing long internodes the mycelium needs to improve its structural support, i.e. its tear-

257 resistance. Long hyphae are at risk of fragmentation by shear-stresses ${ }^{47}$. To deal with this

258 risk, hyphal cell walls can thicken and/or hyphal diameter can increase ${ }^{5,48}$. This is congruent

259 with our finding that long internodes are linked with larger hyphal diameters (IL - D in Fig.2i).

260

261 Conclusion. One of the most fundamental decisions a growing hypha has to make is when

262 to branch. Thus, it is maybe not surprising that internodal length was a highly influential

263 variable (aligned with PC axis 1, Fig. 2) in understanding the architecture of mycelia in trait

264 space. This suggests that the trait internodal length is a main driver of mycelium architecture.

265 Mycelia with short internodes can branch more frequently thus developing a more complex

266 mycelium than those with long internodes. However, the capability of growing long

267 unbranched hyphae enables the mycelium to more flexibly adjust their mycelial modules (see

268 positive correlations between IL and $\left.\mathrm{Db}_{\mathrm{cv}}, \mathrm{L}_{\mathrm{cv}}\right)$ in response to environmental conditions.

269

270 It is interesting that there were no sharp boundaries in the sense of architectural 'syndromes'

271 or clear groups of traits, but rather relatively gradual changes in trait values within the set of

272 fungal isolates we examined. This illustrates the relative versatility of the mycelial growth

273 form in evolutionary terms, at least in the peripheral growth zone of the fungus, which we

274 examined here. We clearly show that there are limits to how a mycelium can be constructed,

275 since some trait combinations are evidently not favorable (e.g. long internodes and small

276 diameters). However, fungi have evidently otherwise filled the trait space within the

277 constraints of such fundamental tradeoffs, even seen in a sample of 31 species. It will be

278 interesting to compare our results to others sets of fungi once such data are available: our

279 results suggest key parameters on which to focus.

280

281 Mycelial architecture is a fundamental property of filamentous fungi, governing the way these

282 organisms explore their substrate. Using a set of fungi co-occurring in the same soil, we

283 show that architectural features vary strongly and reproducibly among different isolates

284 under the same laboratory conditions. It is therefore highly likely that such differences

285 contribute to enabling coexistence within fungal communities ${ }^{49}$ by offering fungi different

286 ways to forage and colonize the soil environment. On the other hand, such trait divergence

287 can also mediate functional complementarity, for example in decomposition or soil

288 aggregation ${ }^{13}$.

289

290 


\section{References}

293 1. Wessels, J. G. H. Fungi in their own right. Fungal Genetics and Biology 27, 134-145

294

295

296

297

298

299

300

301

302

303

304

305

306

307

308

309

310

311

312

313

314

315

316

317

318

319

320

321

322

323

324

325

326

327

328

329

330

331

332

333

334

335

336

337

338

339

340

341

342

343

344

345

(1999).

2. Plomley, N. J. B. Formation of the colony in the fungus Chaetomium. Australian Journal of Biological Science 12, 53-64 (1959).

3. Trinci, A. P. J. A study of the kinetics of hyphal extension and branch initiation of fungal mycelia Journal of Genetic Microbiology 81, 225-236 (1974).

4. Butler, G. M. Growth of hyphal branching systems in Coprinus disseminatus. Annals of Botany 25, 341-\& (1961).

5. McLean, K. M. \& Prosser, J. I. Development of vegetative mycelium during colony growth of Neurospora crassa. Transactions of the British Mycological Society 88, 489495 (1987).

6. Robinson, P. M. \& Smith, J. M. Apical branch formation and cyclic development in Geotrichum candidum. Transactions of the British Mycological Society 75, 233-238 (1980).

7. Barry, D. J. Quantifying the branching frequency of virtual filamentous microbes using fractal analysis. Biotechnology and Bioengineering 110, 437-447 (2013).

8. Moore, D., Robson, G. D. \& Trinci, A. P. J. 97-98 (Cambridge University Press, Cambridge, UK, 2011).

9. Carlile, M. J., Watkinson, S. C. \& Gooday, G. W. (Academic Press, UK, 2001).

10. Katz, D., Goldstein, D. \& Rosenberger, R. F. Model for branch initiation in Aspergillus nidulans based on measurements of growth parameters. Journal of Bacteriology 109, 1097-1100 (1972).

11. Watters, M. K., Lindamood, E., Meunich, M. \& Vetor, R. Strain-dependent eelationship between growth rate and hyphal branching in Neurospora crassa. Proceedings of the Indiana Academy of Science 117, 1-6 (2008).

12. Aguilar-Trigueros, C. A. et al. Branching out: Towards a trait-based understanding of fungal ecology. Fungal Biology Reviews 29, 34-41 (2015).

13. Lehmann, A. \& Rillig, M. C. Understanding mechanisms of soil biota involvement in soil aggregation: A way forward with saprobic fungi? Soil Biology \& Biochemistry $\mathbf{8 8}$, 298-302 (2015).

14. Andrade-Linares, D. R., Veresoglou, S. D. \& Rillig, M. C. Temperature priming and memory in soil filamentous fungi. Fungal Ecology 21, 10-15 (2016).

15. Gams, W. \& Domsch, K. H. Beitrage zur Anwendung der Bodenwaschtechnik für die Isolierung von Bodenpilzen. Arch. Mikrobiol. 58, 134-144 (1967).

16. Thorn, R. G., Reddy, C. A., Harris, D. \& Paul, E. A. Isolation of saprophytic basidiomycetes from soil. Applied and Environmental Microbiology 62, 4288-4292 (1996).

17. Schneider, C. A., Rasband, W. S. \& Eliceiri, K. W. NIH Image to ImageJ: 25 years of image analysis. Nature Methods 9, 671-675 (2012).

18. Posser, J. I. in The growing fungus (eds N.A.R. Gow \& G.M. Gadd) (Springer, UK, 1995).

19. Obert, M., Pfeifer, P. \& Sernetz, M. Microbial growth patterns described by fractal geometry. Journal of Bacteriology 172, 1180-1185 (1990).

20. Zhang, T. Y. \& Suen, C. Y. A fast parallel algorithm for thinning digital patterns. Communications of the Acm 27, 236-239 (1984).

21. Karperien, A. FracLac for ImageJ (1999-2013).

22. Dray, S. On the number of principal components: A test of dimensionality based on measurements of similarity between matrices. Computational Statistics \& Data Analysis 52, 2228-2237 (2008).

23. Chessel, D., Dufour, A. B. \& Thioulouse, J. The ade4 package - I : One-table methods. R News 4, 5-10 (2004).

24. Dray, S. \& Dufour, A. B. The ade4 package: Implementing the duality diagram for ecologists. Journal of Statistical Software 22, 1-20 (2007). 
25. Dray, S., Dufour, A. B. \& Chessel, D. The ade4 package-II: Two-table and K-table methods. $R$ News 7, 47-52 (2007).

26. Diaz, S. et al. The global spectrum of plant form and function. Nature 529, 167-U173 (2016).

27. Duong, T. ks: Kernel smoothing v. R package version 1.11.0. (2018).

28. Kembel, S. W. et al. Picante: R tools for integrating phylogenies and ecology. Bioinformatics 26, 1463-1464 (2010).

29. Paradis, E., Claude, J. \& Strimmer, K. APE: Analyses of Phylogenetics and Evolution in R language. Bioinformatics 20, 289-290 (2004).

30. Cooper, N., Thomas, G. H. \& FitzJohn, R. G. Shedding light on the "dark side' of phylogenetic comparative methods. Methods in Ecology and Evolution 7, 693-699 (2016).

31. Garland, T., Harvey, P. H. \& Ives, A. R. Procedures for the analysis of comparative data using phylogenetically indpendent contrasts. Systematic Biology 41, 18-32 (1992).

32. Freckleton, R. P. \& Harvey, P. H. Detecting non-Brownian trait evolution in adaptive radiations. Plos Biology 4, 2104-2111 (2006).

33. Grafen, A. The phylogenetic regression. Philosophical Transactions of the Royal Society of London Series B-Biological Sciences 326, 119-157 (1989).

34. Jones, K. E. \& Purvis, A. An optimum body size for mammals? Comparative evidence from bats. Functional Ecology 11, 751-756 (1997).

35. Orme, D. The caper package: comparative analysis of phylogenetics and evolution in R v. 1.0 .1 (2013).

36. Wei, T. \& Simko, V. R package "corrplot": Visualization of a Correlation Matrix v. 0.84 (2017).

37. Cade, B. S. \& Noon, B. R. A gentle introduction to quantile regression for ecologists. Frontiers in Ecology and the Environment 1, 412-420 (2003).

38. Cade, B. S., Terrell, J. W. \& Schroeder, R. L. Estimating effects of limiting factors with regression quantiles. Ecology 80, 311-323 (1999).

39. Schloerke, B. et al. GGally: Extension to 'ggplot2' v. 1.3.2 (2017).

40. R Development Core Team. R: A language and environment for statistical computing v. 3.4.1 (2014).

41. Wickham, H. ggplot2: Elegant graphics for data analysis. (Springer, 2009).

42. Henis, Y., Okon, Y. \& Chet, I. Relationship between early hyphal branching and formation of sclerotia in Sclerotium rolfsii. Journal of General Microbiology 79, 147150 (1973).

43. Ho, H. H. Hyphal branching systems in Phytophthora and other Phcomycetes. Mycopathologia 64, 83-86 (1978).

44. Hutchinson, S. A., Sharma, P., Clarke, K. R. \& Macdonald, I. Control of hyphal orientation in colonies of Mucor hiemalis. Transactions of the British Mycological Society 75, 177-191 (1980).

45. Kotov, V., Anishchenko, I., Sirenko, I. \& Reshetnikov, S. Statistical analysis of structural and kinetic characteristics of fungal colony growth with Trichoderma viride Pers.: S.F. Gray. Microbiological Research 160, 273-278 (2005).

46. Trinci, A. P. J. A kinetic study of the growth of Aspergillus nidulans and other fungi. Journal of Genetic Microbiology 57, 11-24 (1969).

47. Suijdam, J. C. \& van Metz, B. Fungal pellet breakup as a function of shear in a fermentor. Journal of fermentation technology 59, 329-333 (1981).

48. Trinci, A. P. J. \& Collinge, A. J. Hyphal wall growth in Neurospora crassa and Geotrichum candidum. Journal of General Microbiology 91, 355-361 (1975).

49. Crowther, T. W. et al. Untangling the fungal niche: the trait-based approach. Frontiers in Microbiology 5 (2014). 
bioRxiv preprint doi: https://doi.org/10.1101/361253; this version posted July 3, 2018. The copyright holder for this preprint (which was not certified by peer review) is the author/funder, who has granted bioRxiv a license to display the preprint in perpetuity. It is made available under aCC-BY-NC-ND 4.0 International license.

\section{Acknowledgements}

401 This work was supported by the Deutsche Forschungsgemeinschaft (RI 1815/16-1). MCR

402 additionally acknowledges an ERC Advanced Grant (694368).

403

404 Conflicts of interest

405 The authors declare no conflicts of interest.

406 
bioRxiv preprint doi: https://doi.org/10.1101/361253; this version posted July 3 , 2018. The copyright holder for this preprint (which was not certified by peer review) is the author/funder, who has granted bioRxiv a license to display the preprint in perpetuity. It is made available under aCC-BY-NC-ND 4.0 International license.

\section{Figure captions}

408

409

410

411

412

413

414

415

416

417

418

419

420

421

Fig. 1 Overview of fungal strains, mycelial image and trait distribution. (a) Phylogenetic tree of the 31 fungal strains belonging to the phyla Ascomycota, Basidiomycota and Mucoromycotina with colony pictures. Pictures are from four-week old cultures grown on potato dextrose agar, and their order follows the order of the phylogenetic tree. Further information about phylogeny and accession numbers of the 31 strains are available in Table S1. (b) An example of mycelial pictures obtained from the setup introduced in the material and method section. Imaged strain is Mucor fragilis. (c) Tukey boxplot of the ten architectural trait variables, mean value and their coefficient of variation (CV) measured in this study: box counting dimension (unitless, $D b$ with $n=8, D b_{C V}$ with $n=8$ ), lacunarity (unitless, $L$ with $n=8, L_{c v}$ with $n=8$ ), branching angle (in ${ }^{\circ}$, BA with $n=5, B A_{C V}$ with $n=5$ ), hyphal diameter (in $\mu \mathrm{m}$, D with $n=5, D_{c v}$ with $n=5$ ), internodal length (in $\mu \mathrm{m}$, IL with $n=5, I L_{c v}$ with $n=5$ ). The boxplots represent 25 th and 75 th percentile, median and outlying points. Information about phylum affiliation is color-coded (black: Mucoromycotina, grey: Basidiomycota, white: Ascomycota).
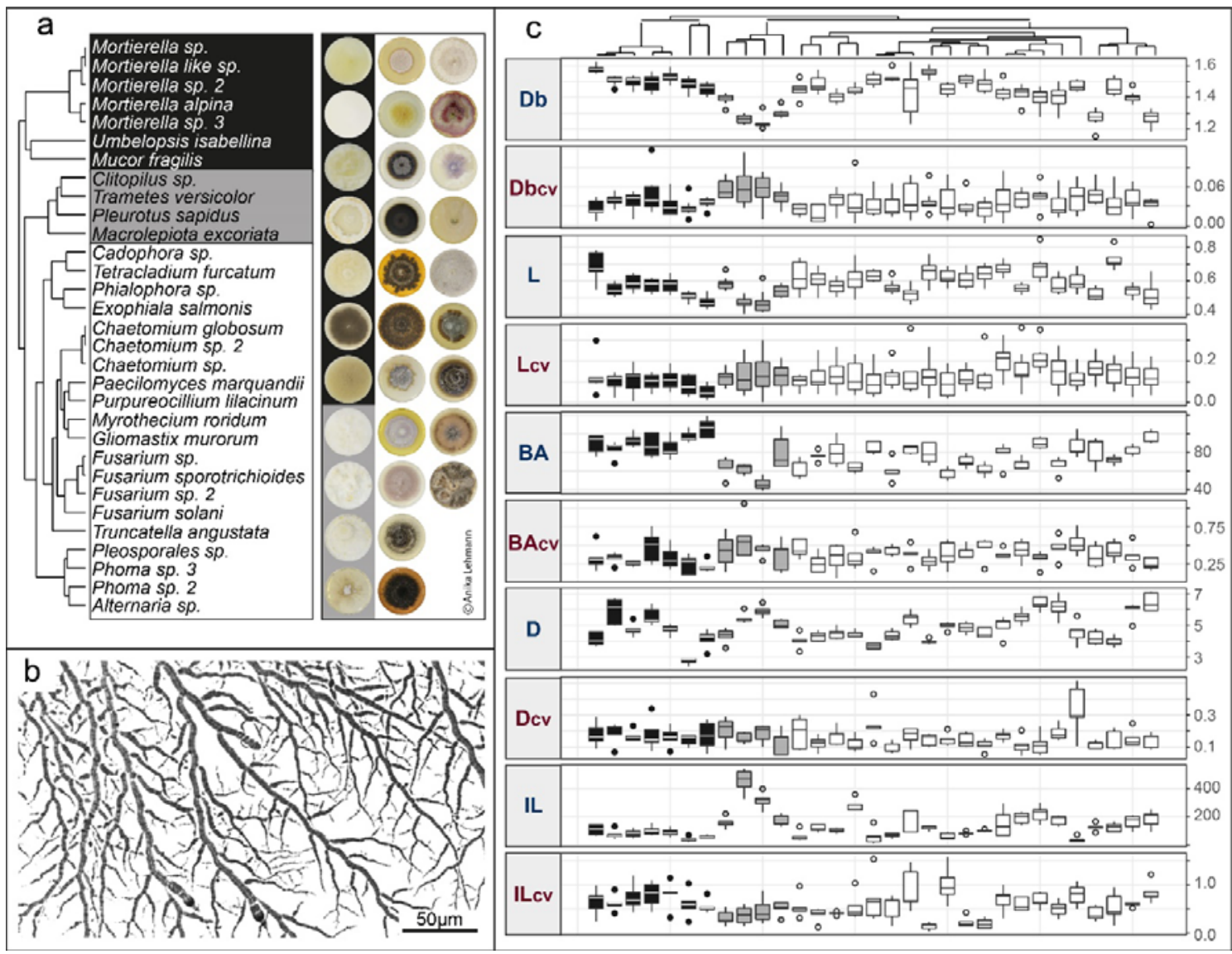
426 Fig. 2 Outcomes of principal component analysis, trait correlation, linear and quantile

427 regression of the investigated architectural traits. Analyses were conducted on trait data $(n=31)$.

428 (a) Projection of the ordinated 31 fungal strains onto ten architectural trait variables (mean and CV):

429 box counting dimension ( $\mathrm{Db}$ ), lacunarity (L), branching angle (BA), hyphal diameter (D), internodal

430 length (IL) into two-dimensional trait space represented by principal component axis 1 and 2

431 (explaining 34 and 18\% of variance, respectively). Arrows indicate direction and weight of trait vectors.

432 Color gradient represents probability of species occurrence (white = low, red = high) in the trait space,

433 with the contour lines denoting the 0.50 and 0.95 quantiles of kernel density estimation (see materials

434 and methods section). (b) Correlation plot of five architectural trait variables and their coefficients of

435 variation. The upper triangle displays original while the lower triangle represents phylogenetically

436 corrected data correlations. Color gradient and square size are proportional to correlation coefficient

437 (Pearson's rho). Asterisks denote significance level: ${ }^{* *}<0.001,{ }^{* *}<0.01,{ }^{*}<0.05$. In grey, we

438 highlight trait combinations affected by detected phylogenetic signal (Table S2). (c-j) The eight

439 strongest trait relationships for either original and/or phylogenetically corrected data. Red lines

440 represent linear regression lines and blue lines quantile regression lines, while line type depicts

441 significance of regression lines; solid lines $p$-value $<0.05$, dashed lines $>0.05$. Corresponding

442 regression statistics can be found in Table S4. Adjusted $R^{2}$ values correspond to linear regressions. 
bioRxiv preprint doi: https://doi.org/10.1101/361253; this version posted July 3, 2018. The copyright holder for this preprint (which was not certified by peer review) is the author/funder, who has granted bioRxiv a license to display the preprint in perpetuity. It is made available under aCC-BY-NC-ND 4.0 International license.
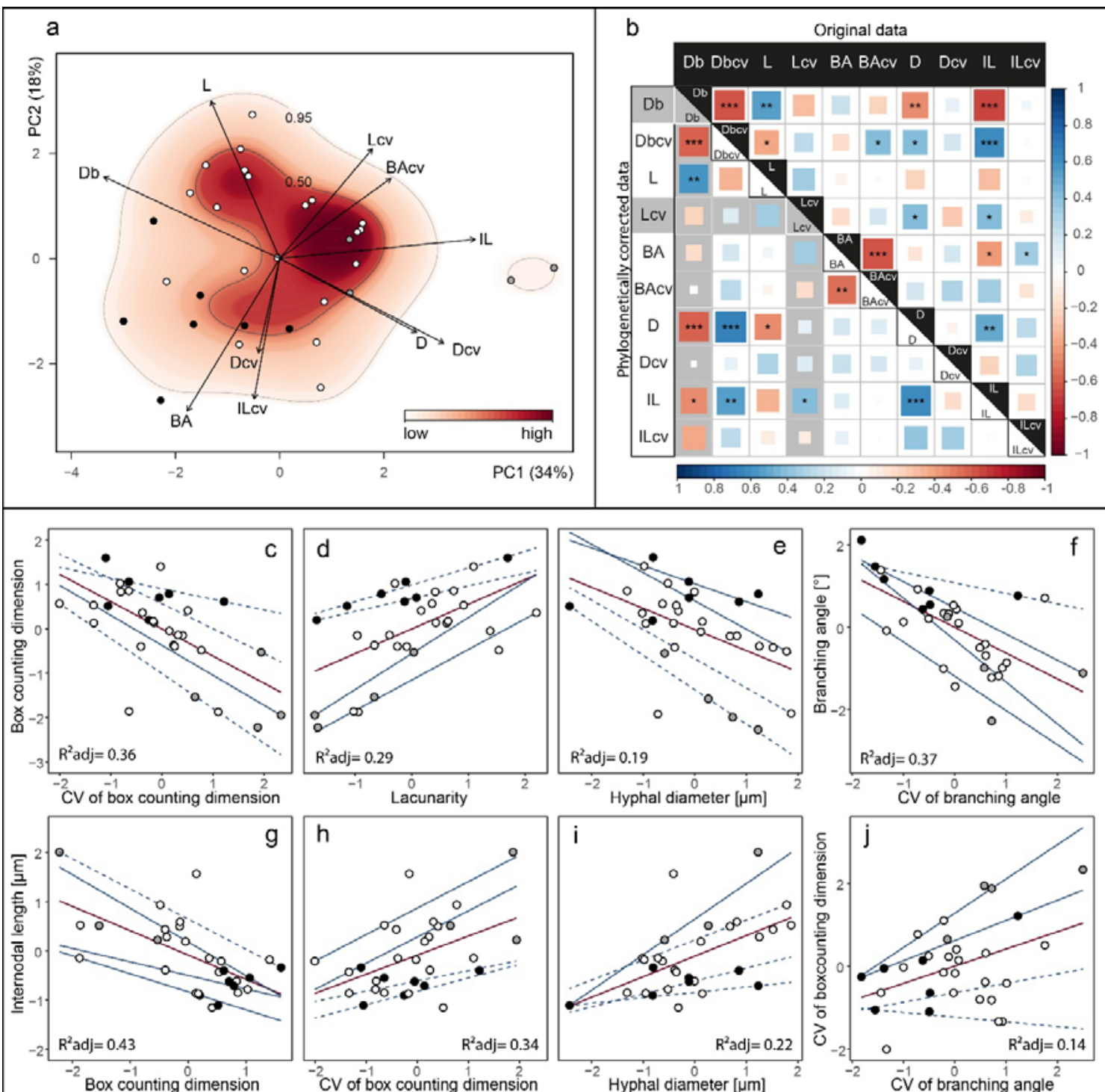